\title{
Association of Baseline Patient-Reported Outcome Measures with Comorbidities in Patients Undergoing Elective Shoulder Arthroscopy
}

\section{Sheena J. Amin MD ${ }^{1 *}$, Steven DeDroda MD², Joseph Gil MD², Mary K. Mulcahey ${ }^{3}$}

${ }^{1}$ University of Missouri-Kansas City, Kansas City, $M O$

${ }^{2}$ Brown University Hospital/Rhode Island Hospital, Providence, RI

${ }^{3}$ Tulane University School of Medicine, New Orleans, LA, Investigation performed at the Department of Orthopedic Surgery, Hahnemann University Hospital, Philadelphia, PA

Received Date: September 12, 2021; Accepted Date: September 21, 2021; Published Date: September 29, 2021;

"Corresponding author: Sheena J. Amin MD, University of Missouri-Kansas City, Kansas City, MO. 732-692-7606, Email: saa322@drexel.edu/sheena614@gmail.com

\section{Abstract}

\section{Introduction}

Shoulder pathology causes debilitating pain and can prevent individuals from participating in their activities of daily living. Following appropriate evaluation and failure of non-operative management, elective shoulder arthroscopy may be performed to alleviate symptoms in these patients. The purpose of this study was to determine the relationship between baseline patient-reported outcome measures (proms) and demographics, social factors, and medical comorbidities in patients undergoing elective shoulder arthroscopy.

\section{Methods}

A retrospective chart review was conducted to collect patient data including demographics, social factors, and medical comorbidities. The American Shoulder and Elbow Surgeons Shoulder Score (ASES) and the Simple Shoulder Test (SST) scores were obtained via preoperative questionnaires. Pearson $r$ correlation was calculated to determine whether individual factors were associated with ASES/SST scores.

\section{Results}

Thirty-four patients were included. The mean age and BMI were 51.5 years and $31.4 \mathrm{~kg} / \mathrm{m}^{2}$ respectively. Average ASES score was $15.5(\mathrm{~N}=24)$. Average SST score was $3.1(\mathrm{~N}=34)$. Smokers (mean 9.8 vs. 20.5 non-smokers, $\mathrm{p}=0.002$ ) and individuals with full (mean 9.9 vs. 19.1 without tear, $\mathrm{p}=0.015$ ) and partial thickness tears (mean 25.8 vs. 14.1 without tear, $\mathrm{p}=0.016$ ) of the supraspinatus tendon had significantly lower ASES scores preoperatively. Hypertension (mean 2.1 with vs. 3.7 without, $\mathrm{p}=0.034$ ) and mental illness (mean 1.4 vs. 3.5 without, $\mathrm{p}=0.014$ ) had a statistically significant impact on preoperative SST scores. Any tear of the four rotator cuff tendons were significantly associated with lower baseline SST scores (mean 2.3 vs. 4.4 without tear, $\mathrm{p}=0.005$ ). The other factors reviewed in this study were not observed to be associated with baseline ASES/SST scores.

\section{Conclusion}

Current smoking status, partial and full thickness rotator cuff tears, hypertension, and mental illness were shown to have a significant impact on baseline shoulder outcome scores, depending on which score was measured. This information may be used to counsel patients at risk for worse outcomes following elective shoulder arthroscopy. Developing a better understanding of the association between medical comorbidities and baseline shoulder outcome scores will allow orthopaedic surgeons to more effectively counsel their patients regarding anticipated postoperative outcomes.

Keywords: Comorbidities; Demographic; Patient reported outcome measures; Shoulder arthroscopy; Social

\section{Introduction}

Patient-reported outcome measures (proms), such as the American Shoulder and Elbow Surgeons (ASES) score and the Simple Shoulder Test (SST) have been validated to assess shoulder function in patients, pre- and postoperatively. The ASES questionnaire is composed of a series of ten questions, asking patients to indicate the ease with which they can perform everyday tasks, such as putting on a coat or combing one's hair [2]. The questionnaire also assesses the patient's ability to perform strenuous tasks, such as their ability to lift ten pounds over their shoulder [2]. In contrast, the SST also provides physicians with a validated shoulder outcome score that assesses the ability to perform tasks, such as tossing a softball with their afflicted shoulder [2]. Studies have shown that SST scores can distinguish patients with pathological conditions of the shoulder from those who do not [2]. Since the test is composed of a series of yes/no questions, SST scores can also help monitor improvement in shoulder function after surgery [2]. In addition, since ASES and SST scores are patient reports, they can evaluate the clinical significance of individual variables.

Investigations have previously examined the relationship between demographic factors and functional shoulder scores for individuals undergoing elective arthroscopic surgery $[\mathbf{3 , 6}$, 10, 12-14]. Li et al. Examined the effect of obesity on ASES scores in patients undergoing total shoulder arthroplasty [3]. The authors found that mean baseline ASES scores for obese (BMI over $40 \mathrm{~kg} / \mathrm{m}^{2}$ ), overweight (BMI of 30 to $39.9 \mathrm{~kg} / \mathrm{m}^{2}$ ), and normal weight (BMI of 25 to $29.9 \mathrm{~kg} / \mathrm{m}^{2}$ ) individuals were 35.8, 37.4, and 38.4, respectively [3]. Despite differences in preoperative shoulder scores, Li et al. Determined that postoperatively all three groups had significantly improved ASES scores $(\mathrm{P}<0.001)$ [3]. In contrast, the authors found that patients with a normal BMI had significant improvement in physical function of the shoulder, as measured by the Short Form 36 (SF-36), while neither of the other two groups 
experienced a similar benefit $[\mathbf{3}, \mathbf{1 4}]$. Oh et al. Evaluated the functional scores (ASES, SST) of 177 patients between age 24 and 80 years who had undergone arthroscopic rotator cuff repair to determine the effect of age on post-operative outcome [6]. There was a significant improvement in ASES and SST scores after surgery, however, there were no significant differences in these scores associated with age [6].

Other studies have examined the impact of comorbidities on baseline shoulder functional scores and post-operative outcome scores in patients with rotator cuff injury $[12,13]$. In 2014, Tashjian et al. Evaluated patients who had undergone surgery for a rotator cuff tear, and they reported that individuals with a greater number of comorbidities had lower baseline scores [12]. For the purpose of their study, they analyzed the impact of age, sex, worker's compensation status, surgical history, smoking history, tear size, symptom duration, and patient expectations on preoperative baseline shoulder scores [12]. The authors conducted a follow-up study in 2016 to evaluate the impact of comorbidities on outcome scores after either open, mini-open, or arthroscopic rotator cuff repair [13]. They determined that patients with a greater number of medical comorbidities had worse outcomes after rotator cuff repair, but that these patients also experienced greater longterm improvement in shoulder function [13].

Although elective arthroscopic shoulder surgery is common and prior studies have investigated the influence of demographics and medical comorbidities on outcome scores following these procedures, there is a paucity of data regarding the relationship between demographics, medical comorbidities, and social factors on preoperative functional scores. A study by Shields et al. Evaluated the relationship between psychiatric illness (e.g. Depression, anxiety, or bipolar disorder) and outcome scores for patients with humeral shaft fractures, and found that patients without psychiatric illness reported better outcome scores [10]. However, there is a lack of information regarding the impact of psychiatric illness on baseline shoulder functional scores for individuals undergoing elective shoulder arthroscopy.

The purpose of this study was to evaluate the relationship between baseline shoulder functional scores and demographics, medical comorbidities, social factors and preoperative imaging findings in order to determine if any of these factors increase the risk of poor baseline shoulder function. We hypothesized that current smokers and patients with medical comorbidities (e.g. Hypertension, mental illness) would have lower baseline proms.

\section{Methods}

Following Institutional Review Board (IRB) approval (protocol \#1701005074), a retrospective chart review was performed. Patients who underwent elective arthroscopic shoulder surgery between July 2014 and September 2016 by a single surgeon at a single institution were included. Final inclusion of patients was determined based on the availability of complete patient demographic information, medical data, ASES/SST functional scores, and preoperative imaging. Patients from special populations, such as adults unable to consent, individuals under the age of 18 , pregnant women, and prisoners were excluded from this study.

Patient demographic information, which included age, sex, height, weight, as well as alcohol, tobacco, and drug usage, was collected at their initial visit. Patients were also provided with yes/no questions about prior medical history on their intake questionnaire. The variables analyzed in this study included alcohol use, smoking, sex, BMI (normal: 18.5 to 24.9 $\mathrm{kg} / \mathrm{m}^{2}$, overweight/obese: $>25 \mathrm{~kg} / \mathrm{m}^{2}$ ), worker's compensation status, arthritis, and mental illness (depression, anxiety, bipolar disorder, and dissociative identity disorder). Baseline shoulder functional scores were determined based on ASES and SST questionnaires, which were completed by patients during their preoperative evaluation. All patients were asked to complete both ASES and SST questionnaires. Diagnostic radiographic imaging was reviewed to evaluate bony anatomy (x-rays) and to determine the type and extent of rotator cuff tear (MRI). Patients were evaluated independently for the presence or absence of each variable.

\section{Statistical Methods}

Pearson $\mathrm{r}$ correlation was calculated in SPSS (IBM Analytics, Armonk, NY) to determine if the observed independent variables were associated with baseline ASES/SST scores. The Pearson $r$ coefficient was used to measure the strength of the relationship between individual variables and baseline shoulder scores. Variables were considered significant if $\mathrm{p}<0.05$.

\section{Results}

Thirty-four patients (16 women and 18 men) met the inclusion criteria for this study. Complete demographic information, medical data, SST questionnaires, and preoperative imaging were available for 34 patients (16 women and 18 men). Ten patients were excluded during evaluation of ASES scores due to incomplete ASES questionnaires. Complete demographic information, medical data, ASES questionnaires, and preoperative imaging were available for 24 patients (11 women and 13 men).

\section{Alcohol}

On review of the ASES questionnaires, 6 out of 24 patients (25\%) were found to consume some amount of alcohol (mean 10.17 vs. 18 non). On review of the SST questionnaires, 11 out of 34 patients $(32.4 \%)$ indicated that they consumed some amount of alcohol (mean 2.45 vs. 3.43 non). Alcohol consumption did not show a significant impact on ASES or SST scores ( $\mathrm{p}=0.067$ and $\mathrm{p}=0.229$ respectively) (Table 1 and 2).

\begin{tabular}{|c|c|c|c|c|}
\hline & Number of Participants $(\mathrm{N}=24)$ & Mean ASES Score & $\mathbf{R}$ & $\mathbf{P}$ \\
\hline \multicolumn{5}{|c|}{ Demographics } \\
\hline \multicolumn{3}{|c|}{ Sex } & -0.182 & 0.393 \\
\hline Female & 11 & 14.27 & & \\
\hline Male & 13 & 17.54 & & \\
\hline \multicolumn{3}{|c|}{ BMI } & 0.221 & 0.299 \\
\hline Obese & 12 & & & \\
\hline Overweight & 10 & & & \\
\hline Obese + Overweight & 22 & 16.64 & & \\
\hline Normal & 2 & 9.5 & & \\
\hline \multicolumn{5}{|c|}{ Social Factors } \\
\hline \multicolumn{3}{|c|}{ Smoking History* } & -0.591 & 0.002 \\
\hline Current & 9 & & & \\
\hline Former & 1 & & & \\
\hline Current + Former & 10 & 9.8 & & \\
\hline Non-Smoker & 14 & 20.5 & & \\
\hline \multicolumn{3}{|c|}{ Alcohol Consumption } & -0.38 & 0.067 \\
\hline Yes & 6 & 10.17 & & \\
\hline
\end{tabular}




\begin{tabular}{|c|c|c|c|c|}
\hline No & 18 & 18 & & \\
\hline \multicolumn{5}{|c|}{ Medical Comorbidities } \\
\hline \multicolumn{3}{|c|}{ HTN } & -0.397 & 0.055 \\
\hline Yes & 12 & 12.5 & & \\
\hline No & 12 & 19.58 & & \\
\hline \multicolumn{3}{|c|}{ Arthritis } & -0.232 & 0.274 \\
\hline Osteoarthritis & 4 & & & \\
\hline Arthritis & 1 & & & \\
\hline $\mathrm{OA}+$ Arthritis & 5 & 12 & & \\
\hline None & 19 & 17.12 & & \\
\hline \multicolumn{3}{|c|}{ Mental Illness } & -0.278 & 0.188 \\
\hline Depression & 1 & & & \\
\hline Depression and Anxiety & 2 & & & \\
\hline Bipolar Disorder & 1 & & & \\
\hline Dissociative Identity Disorder & 1 & & & \\
\hline Total & 5 & 12.2 & & \\
\hline No & 19 & 17.32 & & \\
\hline \multicolumn{5}{|c|}{ Radiographic Imaging Results } \\
\hline \multicolumn{3}{|c|}{$\begin{array}{c}\text { Tear of Rotator Cuff } \\
\text { (Includes partial/full thickness tears of supraspinatus, infraspinatus, teres minor, and } \\
\text { subscapularis tendons) }\end{array}$} & -0.12 & 0.609 \\
\hline Yes & 17 & 15.41 & & \\
\hline No & 7 & 17.57 & & \\
\hline
\end{tabular}

Table 1: Association of demographic factors, social factors, medical comorbidities, and radiographic imaging results with ASES scores.

\begin{tabular}{|c|c|c|c|c|}
\hline & Number of Participants(N=24) & Mean SST Score & $\mathbf{R}$ & $\mathbf{P}$ \\
\hline \multicolumn{5}{|c|}{ Demographics } \\
\hline \multicolumn{3}{|c|}{ Sex } & -0.093 & 0.599 \\
\hline Female & 16 & 2.81 & & \\
\hline Male & 18 & 3.22 & & \\
\hline \multicolumn{3}{|c|}{ BMI } & 0.13 & 0.464 \\
\hline Obese & 19 & & & \\
\hline Overweight & 11 & & & \\
\hline Obese + Overweight & 30 & 3.13 & & \\
\hline Normal & 4 & 2.25 & & \\
\hline \multicolumn{5}{|c|}{ Social Factors } \\
\hline \multicolumn{3}{|c|}{ Smoking History } & -0.203 & 0.25 \\
\hline Current & 13 & & & \\
\hline Former & 5 & & & \\
\hline Current + Former & 18 & 2.61 & & \\
\hline Non-Smoker & 16 & 3.5 & & \\
\hline \multicolumn{3}{|c|}{ Alcohol Consumption } & -0.212 & 0.229 \\
\hline Yes & 11 & 2.45 & & \\
\hline No & 23 & 3.43 & & \\
\hline \multicolumn{5}{|c|}{ Medical Comorbidities } \\
\hline \multicolumn{3}{|c|}{ HTN $^{*}$} & -0.364 & 0.034 \\
\hline Yes & 15 & 2.13 & & \\
\hline \multirow{2}{*}{\multicolumn{3}{|c|}{$\begin{array}{c}19 \\
\text { Arthritis }\end{array}$}} & & \\
\hline & & & -0.069 & 0.698 \\
\hline Osteoarthritis & 7 & & & \\
\hline Arthritis & 2 & & & \\
\hline $\mathrm{OA}+$ Arthritis & 9 & 2.78 & & \\
\hline None & 25 & 3.12 & & \\
\hline \multicolumn{3}{|c|}{ Mental Illness* } & -0.419 & 0.014 \\
\hline Depression & 2 & & & \\
\hline Depression and Anxiety & 3 & & & \\
\hline Bipolar Disorder & 2 & & & \\
\hline Dissociative Identity Disorder & 1 & & & \\
\hline Total & 8 & 1.38 & & \\
\hline No & 28 & 3.54 & & \\
\hline \multicolumn{5}{|c|}{ Radiographic Imaging Results } \\
\hline \multicolumn{3}{|c|}{$\begin{array}{c}\text { Tear of Rotator Cuff (Includes partial/full thickness tears of supraspinatus, infraspinatus, } \\
\text { teres minor, and subscapularis tendons) }\end{array}$} & -0.468 & 0.005 \\
\hline Yes & 22 & 2.27 & & \\
\hline No & 12 & 4.42 & & \\
\hline
\end{tabular}

Table 2: Association of demographic factors, social factors, medical comorbidities, and radiographic imaging results with SST scores. 


\section{BMI}

For the 24 patients who had completed the ASES questionnaires, the mean BMI was $31.35 \mathrm{~kg} / \mathrm{m}^{2}$ (min=22.8 $\mathrm{kg} / \mathrm{m}^{2}, \max =41.28 \mathrm{~kg} / \mathrm{m}^{2}$ ). Twenty-two out of 24 patients $(91.7 \%)$ undergoing elective arthroscopic shoulder surgery were overweight/obese $\left(\mathrm{BMI}>25 \mathrm{~kg} / \mathrm{m}^{2}\right)$ and had lower ASES scores compared to their normal weight counterparts (mean 16.64 vs. 9.5 normal BMI, $\mathrm{p}=0.299$ ). Of the 34 patients with complete SST questionnaires, $30 \quad(88.2 \%)$ were overweight/obese and this group had lower SST scores compared to normal weight patients (mean 3.13 vs. 2.25 normal BMI, $\mathrm{p}=0.464)$. The mean BMI for these individuals was $32.63 \mathrm{~kg} / \mathrm{m}^{2}\left(\min =20.49 \mathrm{~kg} / \mathrm{m}^{2}, \max =56.98 \mathrm{~kg} / \mathrm{m}^{2}\right)$. Statistical analysis did not show an association between BMI and ASES/SST scores ( $\mathrm{p}=0.299$ and $\mathrm{p}=0.464$ respectively) (Table 1 and 2).

\section{Smoking}

Ten of twenty-four patients $(41.7 \%)$ who completed ASES scores were smokers (mean 9.8 vs. 20.5 non). Of the ten smokers, nine were current smokers $(37.5 \%)$, while one was a former smoker (4.2\%). Smoking had a significant association with lower baseline ASES scores $(r=-0.591, n=24, p=0.002)$ (Table 1), however, smoking was not significantly associated with low SST scores $(\mathrm{p}=0.25)$ (Table 2). Eighteen of 34 patients $(52.9 \%)$ who completed SST evaluations were smokers (mean 2.61 vs. 3.5 non), 5 of whom were former smokers $(14.7 \%)$, and 13 were current smokers $(38.2 \%)$.

\section{Sex}

The ASES group consisted of 11 females (45.8\%) and 13 males (54.2\%) (Mean 14.27 women vs. 17.54 men). The SST group included 16 females $(47.1 \%)$ and 18 males $(52.9 \%)$ (Mean 2.81 females vs. 3.22 males). There was no significant difference between ASES/SST scores and sex $(\mathrm{p}=0.393$ and $\mathrm{p}=0.599$ respectively) (Table 1 and 2).

\section{Arthritis}

Of the 24 patients who completed ASES questionnaires, 4 (16.7\%) had osteoarthritis (OA) (mean ASES=8.25) and one indicated a history of arthritis (ASES $=11$ ), but did not specify OA versus rheumatoid arthritis (RA). There was no significant association between baseline ASES scores and a history of arthritis/OA (mean 12 vs. 17.12 non, $\mathrm{p}=0.274$ ). Seven of the 34 patients $(20.6 \%)$ (mean $\mathrm{SST}=2$ ) who completed SST questionnaires, reported a history of OA while two patients $(5.9 \%)$ indicated a history of arthritis (mean SST=5.5), but did not specify OA versus RA. There was no significant association between SST scores and arthritis/OA (mean 2.78 vs. 3.12 non, $\mathrm{p}=0.698$ ) (Table 1 and 2).

\section{Hypertension (HTN)}

Fifteen of the 34 patients $(44.1 \%)$ in this study had a history of HTN. Twelve of 24 patients $(50 \%)$ who completed ASES evaluations had HTN (mean 12.5 vs. 19.58 non). There was no significant association between HTN and ASES scores $(p=0.055)$ (Table 1). Fifteen of $34(44.1 \%)$ patients who completed the SST questionnaire had hypertension (mean 2.13 vs. 3.74 non). HTN had a significant impact on baseline SST scores $(\mathrm{r}=-0.364, \mathrm{n}=34, \mathrm{p}=0.034)$, correlating with lower shoulder function (Table 2).

\section{Mental Illness}

ASES/SST scores for patients with a history of depression, anxiety, bipolar disorder, and dissociative identity disorder were compared to patients who did not report a history of mental illness. Five of 24 patients $(20.8 \%)$ who completed the ASES questionnaire were identified as having a history of a psychiatric disorder, which was not shown to have a significant association with preoperative ASES scores (mean
12.2 vs. 17.32 non; $r=-0.278, n=24, p=0.188$ ) (Table 1). Eight of 34 patients $(23.5 \%)$ who completed the SST questionnaire had a history of mental illness. Patients with mental illness were found to have significantly lower SST scores than patients without mental illness (mean 1.38 vs. 3.54 non; $r=-$ 0.419, $\mathrm{n}=34, \mathrm{p}=0.014$ ) (Table 2).

\section{Rotator Cuff Tears}

Seventeen of the 24 patients $(70.1 \%)$ who completed the ASES questionnaire had tears ot the rotator cuff. Of those individuals, 12 patients had tears of the supraspinatus tendon, which is the most common tendon injured. Rotator cuff tears did not have a significant correlation with baseline ASES scores (mean ASES 15.41 vs. 17.57 non; $\mathrm{r}=-0.12$, $\mathrm{n}=24$, $\mathrm{p}=0.609$ ) (Table 1). However, patients with rotator cuff tears did have lower, although not statistically significant, ASES score when compared to patients without tears. Twenty-two of the 34 patients $(64.71 \%)$ who completed the SST questionnaire, had a rotator cuff tear. Rotator cuff tears were shown to have a statistically significant association with lower baseline SST scores (mean SST score in patients with tear 2.27 vs. 4.42 without; $\mathrm{r}=-0.468, \mathrm{n}=34, \mathrm{P}=0.005$ ) (Table 2).

\section{Discussion}

This study demonstrated that smoking was associated with lower baseline ASES scores while mental illness, hypertension, and rotator cuff tears were associated with lower baseline SST scores in patients undergoing elective shoulder arthroscopy. Elective shoulder arthroscopy has been shown to be effective in alleviating pain and improving functional outcomes for patients with pathological conditions of the shoulder. Previous studies have evaluated the impact of medical comorbidities and demographics on baseline shoulder outcome scores and demonstrated that factors, such as obesity and psychological distress are associated with lower baseline scores $[3,5,6,8-$ 10, 12-14].

Smokers are more likely to develop shoulder pathology including larger rotator cuff tears than non-smokers [1]. Smoking alters tendons at the molecular level, which has been associated with a greater degeneration of the tendon as well as a reduced healing capacity [4]. In 2016, Matsen et al. Evaluated 339 patients undergoing shoulder arthroplasty to identify factors that could be associated with positive patient outcomes. The authors determined that smoking was significantly associated with lower SST scores during univariate and multivariate analysis [5]. The results of the current study indicate that smoking had a significant impact on baseline ASES scores, corroborating results of past studies, which have demonstrated that smokers experience more pain and reduced shoulder function in comparison to their nonsmoking counterparts $[\mathbf{1 , 4 , 9}]$.

Mental illness was also significantly associated with lower baseline SST scores in this study. In 2014, Potter et al. Evaluated patients who underwent hip arthroscopy to alleviate symptoms associated with femoroacetabular impingement [7]. Patients were given a questionnaire to assess psychological distress in order to determine if there was an association with this condition and baseline hip outcome scores [7]. The authors found that psychological distress was associated with lower patient self-reported baseline hip outcome [7]. This relationship was further supported by Roh et al. Who examined the effect of psychological distress on self-reported outcome scores in patients with pathological shoulder conditions, including adhesive capsulitis, arthritis, rotator cuff disorders, and calcific tendonitis [8]. The authors utilized the Constant-Murley scale, SST, as well as the Disabilities of the Arm, Shoulder, and Hand (DASH) questionnaire to evaluate outcomes, while psychological distress was measured using the Pain Anxiety Symptom Scale (PASS) [8]. Based on their findings, psychological distress was associated with lower SST and DASH outcome scores, but not with Constant-Murley scores. This is in line with findings in the current study, which 
demonstrated that mental illness had a significant association with lower baseline SST scores, but did not have a similar relationship with ASES scores.

\section{Limitations}

There are several limitations to this study. First, only a small number of patients met all of the inclusion criteria and the numbers were further reduced due to incomplete ASES questionnaires. Therefore, although alcohol consumption, BMI, sex, and arthritis did not have a statistically significant association with lower baseline shoulder scores in this study, they should be further examined in future studies with a larger sample size. These variables can affect patient outcomes in a clinically significant way, so certain patient populations may demonstrate worse shoulder function. Second, most patients included in this study had a BMI of over $25 \mathrm{~kg} / \mathrm{m}^{2}$, indicating that they were either overweight or obese. The predominance of overweight/obese patients in this study raises the question of whether patients in this category are more likely to undergo elective shoulder arthroscopy than normal weight individuals with the same shoulder pathology. In addition, the results showed that obese and overweight patients had greater, although not statistically significant, ASES and SST scores than patient with a normal BMI (mean ASES: 16.64 vs. 9.5 normal BMI; mean SST: 3.13 vs. 2.25 normal BMI). However, previous studies have shown that baseline shoulder functional scores are lower for individuals with a higher BMI since obese patients are known to have a higher rate of post-operative complications [3]. This discrepancy in patient functional scores may be due to the small sample size of patients with a normal BMI in this study. Typically, patients with normal BMI $\left(18.5\right.$ to $\left.24.9 \mathrm{~kg} / \mathrm{m}^{2}\right)$ have better shoulder function than those who are overweight/obese $\left(>25 \mathrm{~kg} / \mathrm{m}^{2}\right)$.

Future studies can also be expanded to include to quantitatively evaluate patient outcomes with regards to the relationship between tear size and baseline shoulder ASES/SST scores. This study found that rotator cuff tears were associated with statistically significant lower SST scores. However, results reported in a study by Sugaya et al. showed that larger rotator cuff tears have worse surgical outcomes and are more likely to tear again [11]. Other medical comorbidities, such as diabetes and hyperlipidemia can also be included to study their association with baseline PROM. Additionally, the study can also be expanded to include postoperative PROM to better understand the association of demographic, social, and medical comorbidities with patient outcomes. By determining the relationship between these risk factors and baseline shoulder outcome scores, orthopaedic surgeons may better counsel patients preoperatively regarding the relationship between medical comorbidities, shoulder pathology and overall shoulder function.

\section{Conclusion}

Current smoking status, hypertension, and mental illness were shown to have a significant impact on baseline shoulder outcome scores, depending on which score was measured. This information may be used to educate patients in regards to the relationship between certain risk factors and shoulder pathology. Developing a better understanding of the association between medical comorbidities and baseline shoulder outcome scores will allow orthopaedic surgeons to more effectively counsel their patients regarding anticipated postoperative outcomes.

\section{References}

1. Bishop JY, Santiago-Torres JE, Rimmke N, Flanigan DC (2015) Smoking predisposes to rotator cuff pathology and shoulder dysfunction: a systematic review. Arthroscopy. 31:1598-1605.

2. Kirkley A, Griffin S, Dainty K (2003) Scoring systems for the functional assessment of the shoulder. Arthroscopy. 19:1109-1120.

3. Li X, Williams PN, Nguyen JT, Craig EV, Warren RF et al. (2013) Functional outcomes after total shoulder arthroplasty in obese patients. J Bone Joint Surg Am. 95:160.

4. Lundgreen K, Lian OB, Scott A, Nassab P, Fearon A et al. (2014) Rotator cuff tear degeneration and cell apoptosis in smokers versus nonsmokers. Arthroscopy. 30:936-41.

5. Matsen III FA, Russ SM, Vu PT, Hsu JE, Lucas RM et al. (2016) What factors are predictive of patient-reported outcomes? A prospective study of 337 shoulder arthroplasties. Clin Orthop Rel Res. 474:2496-2510.

6. Oh J, Kim SH, Kang JY, Oh CH, Gong HS (2010) Effect of age on functional and structural outcome after rotator cuff repair. Am J Sports Med. 38:672-678.

7. Potter MQ, Wylie JD, Sun GS, Beckmann JT, Aoki SK (2014) Psychologic distress reduces preoperative selfassessment scores in femoroacetabular impingement patients. Clin Orthop Rel Res. 472:1886-1892.

8. Roh YH, Noh JH, Oh JH, Baek GH, Gong HS (2012) To what degree do shoulder outcome instruments reflect patients' psychologic distress? Clin Orthop Rel Res. 470:3470-3477.

9. Santiago-Torres J, Flanigan DC, Butler RB, Bishop JY (2015) The effect of smoking on rotator cuff and glenoid labrum surgery: a systematic review. Am J Sports Med. 43:745-751.

10. Shields E, Sundem L, Childs S et al. (2015) Factors predicting patient-reported functional outcome scores after humeral shaft fractures. Injury. 46:693-698.

11. Sugaya H, Maeda K, Matsuki K, Moriishi J (2007) Repair integrity and functional outcome after arthroscopic double-row rotator cuff repair: a prospective outcome study. J Shoulder Elbow Surg. 16:41-42.

12. Tashjian RZ, Henn RF, Kang L, Green A (2004) The effect of comorbidity on self-assessed function in patients with a chronic rotator cuff tear. J Bone Joint Surg. 86:355362.

13. Tashjian RZ, Henn RF, Kang L, Green A (2006) Effect of medical comorbidity on self-assessed pain, function, and general health status after rotator cuff repair. J Bone Joint Surg.88:536-540.

14. Yilmaz F, Sahin F, Ergoz E et al. (2008) Quality of life assessments with SF 36 in different musculoskeletal diseases. Clinical Rheumatology. 27:327-33. 